subsequent urine analysis demonstrated glyceroluria and white cell enzyme analysis confirmed the diagnosis of Fructose 1,6 bisphosphatase deficiency in all. Lack of appropriate screening resulted in 4/5 patients presenting with repeated episodes of decompensation with an average of 3.4 episodes per patient. In these patients, average time period between initial presentation and diagnosis with Fructose 1,6 bisphosphatase deficiency was 13.25 months.

Conclusion Our case series emphasise the importance of obtaining urine for organic acid analysis at the time of hypoglycaemia and/or lactic acidosis to avoid delay in diagnosis and initiation of appropriate therapy. Timely diagnosis and treatment can prevent morbidity and potential mortality associated with repeated episodes of decompensation.Fructose 1,6 bisphosphatase deficiency is a treatable metabolic disorder that can be identified with prominent glyceroluria during an acute episode; which might not be seen after recovery from illness. It is important to be aware of false positive results due to contamination from nappy creams and to ensure repeat organic acid analysis in these situations.Management involves avoidance of prolonged fasting and emergency regimen during illnesses which includes administration of glucose polymer and avoiding drinks with higher fructose content.

\section{G473 PRIMARY CARNITINE DEFICIENCY: VARIABLE IN ITS PRESENTATION, TREATABLE IN ITS COURSE AND THE IMPORTANCE OF FAMILY SCREENING. A CASE SERIES}

S Grunewald, O Lewis. Paediatric Metabolic Medicine, Great Ormond Street Hospital, London, UK

\subsection{6/archdischild-2020-rcpch.406}

Background Primary carnitine deficiency (PCD) is an autosomal recessive disorder of fatty acid oxidation, caused by mutations in the SLC22A5 gene encoding for the carnitine transporter OCTN2. Carnitine uptake deficiency prevents the body from using certain fats for energy, particularly during periods of fasting or illness. The first manifestation of PCD can be at any age, where patients can present with hypoglycemia, hyperammonaemia, encephalopathy, skeletal myopathy and cardiomyopathy.

Case Review We report on a previously well 15 year old boy who participated in a challenging Duke of Edinburgh expedition. The following morning he was found drowsy and subsequently admitted to hospital with worsening encephalopathy, hypoglycaemia and hyperammonaemia. This, along with rising CK and troponin levels meant PCD was included in the early differential and carnitine supplementation was commenced. Unfortunately his cardiac failure was too advanced and he had a cardiac arrest, dying after an hour of CPR. Genetic testing confirmed the diagnosis of PCD. On family cascade screening his asymptomatic sister was also diagnosed with PCD, started on oral carnitine supplements and continues to be well.

A retrospective analysis of a total of thirteen diagnosed patients from one centre illustrates the variability of first presentation of PCD. Five children (ranging between 2 months and 7 years) presented with cardiac symptoms; four with cardiomyopathy, one with a cardiac arrest. Two children presented between 1 year and 3 years with hypoketotic hypoglycaemia. Two children presented with muscle fatigability at 7 months and 7 years of age. Another presented at 10 months old with motor regression, failure to thrive and an abnormal ECG. Two children were diagnosed at birth, either by newborn or sibling screening.

Conclusion This case series illustrates that PCD can present at any age with variable symptoms. Although rare, PCD should always be considered in patients with unexplained symptoms including encephalopathy. It is potentially lethal, but also extremely treatable, as early carnitine supplementation equates to excellent prognosis. Lastly, our case highlights the importance of family screening in preventing potential fatalities.

\section{G475(P) CENANI-LENZ SYNDACTYLY IN SIBLINGS WITH A NOVEL HOMOZYGOUS LRP4 MUTATION AND RECURRENT HYPOGLYCAEMIA}

${ }^{1}$ E Steel, ${ }^{2} \mathrm{~J}$ Hurst, ${ }^{3} \mathrm{~T}$ Cullup, ${ }^{4} \mathrm{~A}$ Calder, ${ }^{5} \mathrm{~B}$ Sivakumar, ${ }^{6} \mathrm{P}$ Shah, ${ }^{2} \mathrm{~L}$ Wilson. ${ }^{1}$ Paediatrics, Central Lancashire Teaching Hospitals, Preston, UK; ${ }^{2}$ Clinical Genetics, Great Ormond Street Hospital, London, UK; ${ }^{3}$ Molecular Genetics, Great Ormond Street Hospital, London, UKi ${ }^{4}$ Radiology, Great Ormond Street Hospital, London, UK; ${ }^{5}$ Plastic Surgery, Great Ormond Street Hospital, London, UK; ${ }^{6}$ Endocrinology, Great Ormond Street Hospital, London, UK

\subsection{6/archdischild-2020-rcpch.407}

Cenani-Lenz Syndactyly (CLS) is a rare autosomal recessive syndrome characterized by disorganized oligosyndactyly of upper and lower limbs as well as radioulnar synostosis. Structural renal abnormalities are also common. We report two affected brothers, born to orthodox Jewish parents, in whom we found a novel homozygous missense variant c.4910G >A; p.(Cys1637Tyr) in LRP4 situated in an EGFlike domain between the 4th beta-propeller and transmembrane domains. Both brothers have had recurrent ketotic hypoglycaemia which has not been associated previously. We present 3D-CT imaging illustrating the limb abnormalities in detail.

\section{G476(P) AMINOACYLASE 1 DEFICIENCY, A CLINICAL PROSPECT}

N KamarusJaman, J Davison, P Gissen, S Krywawych. Metabolic Medicine, Great Ormond Street Hospital, London, UK

\subsection{6/archdischild-2020-rcpch.408}

Background Aminoacylase-1 deficiency(OMIM 609924) is a rare form of inborn error of metabolism ( IEM) (Van Coster et al.2005 Sass et al. 2006) inherited by autosomal pattern characterized by increased urinary excretion of specific $\mathrm{N}$ acetyl amino acids.Most patients demonstrate neurologic abnormalities such as intellectual disability, seizures, hypotonia, and motor delay. In this case series we describe four patients from diverse ethnic origins with slightly variable clinical phenotypes.

Methods/Case Report This retrospective case note review was conducted in a single metabolic centre at a tertiary care hospital in the UK. Clinical, biochemical, molecular genetic and neuro-imaging parameters were gathered from the clinical records, with a focus on the neurologic symptoms and signs.

Results Four patients were identified. Three were female in the age between 4-17 years at time of review and one male 